\title{
Effect of Molecular Weight on the Ion Transport Mechanism in Polymerized Ionic Liquids
}

Fei Fan ${ }^{1 *}$, Weiyu Wang ${ }^{2}$, Adam P. Holt ${ }^{3}$, Hongbo Feng ${ }^{1}$, David Uhrig ${ }^{2}$, Xinyi Lv ${ }^{1}$, Tao Hong ${ }^{1}$, Yangyang Wang ${ }^{2}$, Nam-Goo Kang ${ }^{1}$, Jimmy Mays ${ }^{1}$, Alexei P. Sokolov ${ }^{1,3,4}$

${ }^{1}$ Department of Chemistry, University of Tennessee, Knoxville, TN 37996, United States

${ }^{2}$ Center for Nanophase Materials Sciences, Oak Ridge National Laboratory, Oak Ridge, Tennessee 37831, United States

${ }^{3}$ Department of Physics and Astronomy, University of Tennessee, Knoxville, TN 37996, United States

${ }^{4}$ Chemical Sciences Division, Oak Ridge National Laboratory, Oak Ridge, TN 37831, United States

1. Polymerization Synthesis Details and Results
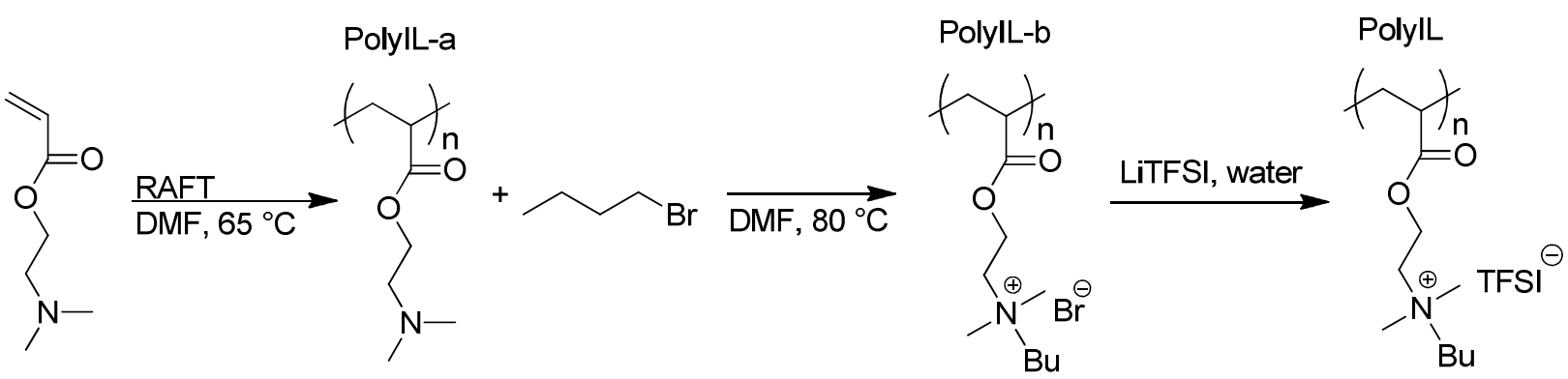

Scheme 4 Synthetic route for polymerized ionic liquids.

PolyIL-a was synthesized using RAFT techniques. Poly-72 and 109 were synthesized using a solid chain transfer agent (CTA), while PolyIL-10 and PolyIL-333 were synthesized using a liquid CTA to achieve better mixing with other reagents. RAFT chain transfer agents: 2(Dodecylthiocarbonothioylthio)-2-methylpropionic acid N-hydroxysuccinimide ester (yellow powder), and Methyl 2-(dodecylthiocarbonothioylthio)-2-methylpropionate (yellow liquid). The resulting structures, with end groups, are presented below. 
PolyIL-Liquid CTA

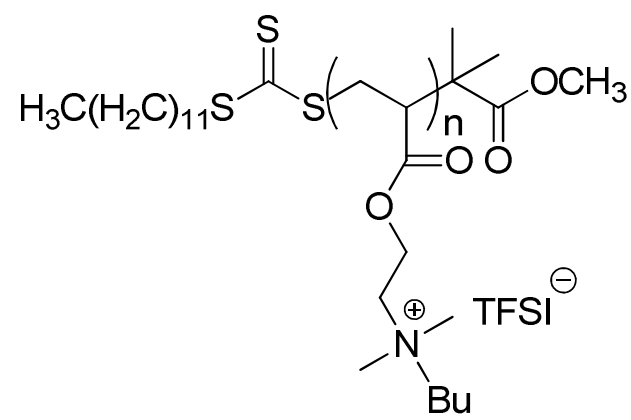

(a)
PolylL-Solid CTA<smiles>CC[C@H](C)SC(=S)[SH](C)CC(C(=O)OCC[N+](C)(Cc1ccccc1)C(F)(F)F)C(C)(C)C(=O)ON1C(=O)CCC1=O</smiles>

(b)

Figure S1 PolyIL structures with end groups, a) PolyIL synthesized using liquid CTA, b) PolyIL synthesized using solid CTA.

The MW of one repeating unit is $480.15 \mathrm{~g} \mathrm{~mol}^{-1}$, and the MW of the end groups is relatively bulky, with liquid CTA of MW $=378.66 \mathrm{~g} \mathrm{~mol}^{-1}$ and solid CTA of $\mathrm{MW}=461.7 \mathrm{~g} \mathrm{~mol}^{-1}$.

2. Master Curves Constructed by Time-Temperature Superposition Principles
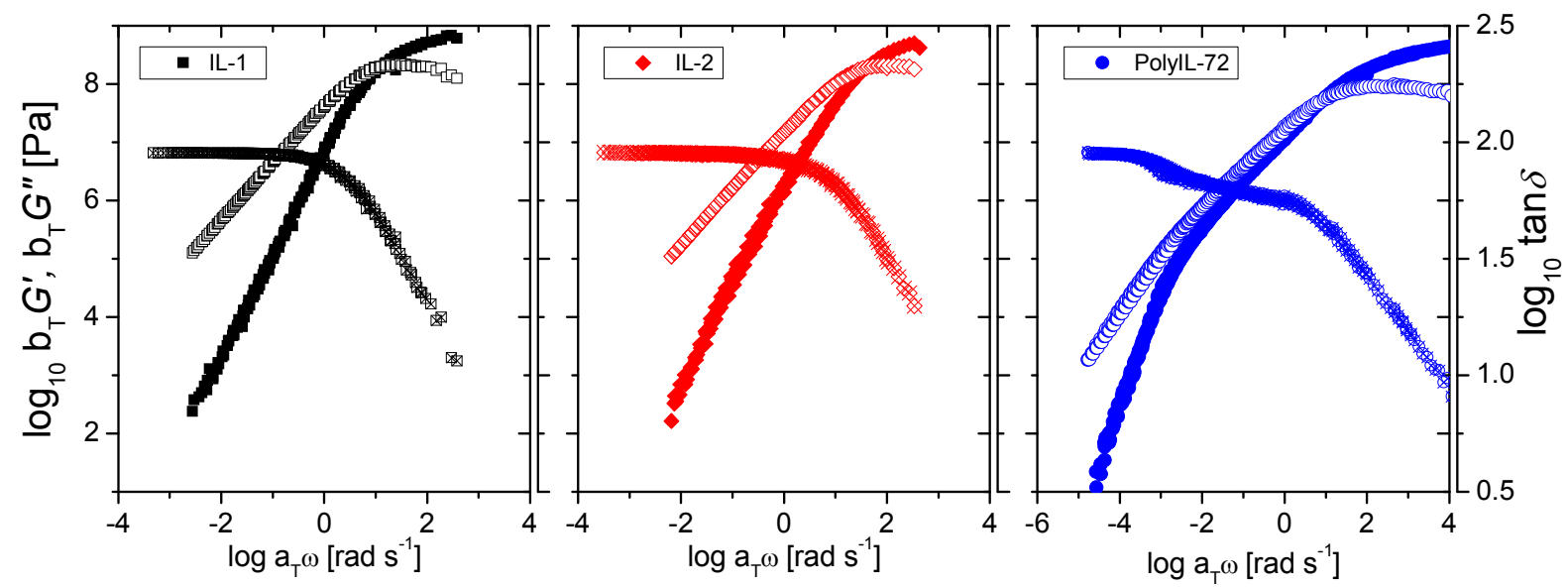

Figure S2. Storage and loss moduli (labeled as $\mathrm{G}^{\prime}$ and $\mathrm{G}^{\prime \prime}$ near each curve) for all the samples, together with tan delta. Master curves are constructed by using time-temperature superposition referenced to $210.6 \mathrm{~K}$ for IL-1, 235.6K for IL-2, 288.1K for PolyIL-72. Tan delta master plot shows that the time-temperature superposition principle works well in the temperature range studied. 
3. Fitting Parameters for Figs. 4 and 6

Table S1 VFT Fit Parameters for Viscosity Values in Fig. $4\left(\mathrm{~T}_{\mathrm{g}} \eta \sim 10{ }^{11} \mathrm{P}\right)$

\begin{tabular}{ccccc}
\hline & $\eta_{0}$ & $B$ & $T_{0}$ & $T_{g}$ \\
\hline \hline IL-1 & -5.0 & 1422.42 & 160.57 & 199.2 \\
IL-2 & -3.667 & 1163.71 & 188.40 & 222.9 \\
IL-3 & -3.017 & 1062.73 & 201.51 & 234.4 \\
PolyIL-10 & -2.098 & 1031.61 & 230.63 & 264.8 \\
PolyIL-72 & -2.187 & 1441.76 & 226.67 & 274.1 \\
PolyIL-109 & -1.918 & 1463.32 & 227.34 & 276.5 \\
PolyIL-333 & -1.607 & 1475.45 & 227.37 & 278.2 \\
\hline \hline
\end{tabular}

Table S2 VFT Fit Parameters for Structural/Segmental Relaxation in Fig.7

\begin{tabular}{ccccccc}
\hline \hline & & $\tau_{0}$ & $B$ & $T_{0}$ & $T_{g}$ & \\
\hline \hline \multirow{3}{*}{ IL-1 } & DLS & -13.707 & 1480.108 & 158.69 & 201.7 & \\
& BDS-1 & -13.343 & 1519.3 & 158.69 & 201.7 & \\
& BDS- $M ”$ & -14.398 & 1699.923 & 155.146 & 200.2 & \\
& Rheology & -14.199 & 1581.189 & 157.862 & 197.8 & $\tau=1000 s$ \\
\hline \multirow{2}{*}{ IL-2 } & BDS-1 & -9.549 & 784.503 & 195.76 & 225.3 & \\
& BDS- $M ”$ & -12.119 & 1062.256 & 191.438 & 224.1 & \\
& Rheology & -11.213 & 882.989 & 195.668 & 222.6 & $\tau=1000 s$ \\
\hline \multirow{2}{*}{ PolyIL- } & BDS1 & -14.894 & 2822.825 & 176.265 & 271.7 & cross over \\
& BDS- $M{ }^{\prime \prime}$ & -14.280 & 2197.75 & 192.94 & 271.9 & point \\
& Rheology & -9.69 & 927.832 & 242.65 & 274.4 & $\tau=1000 s$ \\
\hline \hline
\end{tabular}

4. Calculation of MWs and Molar Conductivity

The MWs of PolyIL- $n$ were calculated based on the $M_{\mathrm{n}}$ of PolyIL- $n$ a.

$$
n=\frac{M_{n} \text { of PolyILna }-M W \text { of end groups }}{(M W \text { of one non }- \text { quaternized repeating unit })}
$$




$$
M W(\text { PolyIL }-n)=(M W \text { of one repeating unit }) \times n+M W \text { of end groups }
$$

$\Lambda=\sigma / c$ ( $\mathrm{c}$ is the concentration of the repeating units), density is assumed to be $1.4 \mathrm{~g} / \mathrm{cm}^{3}$ for all the samples.

IL-1: $c=\frac{1.4 \mathrm{~g} / \mathrm{cm}^{3}}{M W \mathrm{~g} / \mathrm{mol}}$

$\mathrm{IL}-2,3: c=\frac{1.4 \mathrm{~g} / \mathrm{cm}^{3}}{\left(\frac{M W \mathrm{~g} / \mathrm{mol}}{n}\right)}$

PolyIL-10, 72, 109, 333: $c=\frac{1.4 \mathrm{~g} / \mathrm{cm}^{3}}{M W \frac{\mathrm{g}}{\mathrm{mol}} \text { of one repeating unit }}$

\section{XPS Measurement and Results}

X-ray photoelectron spectroscopy (XPS) was performed using a Thermo Scientific Model KAlpha XPS instrument. The instrument uses a monochromated, micro-focusing, $\mathrm{Al} \mathrm{K}_{\alpha} \mathrm{X}$-ray source $(1486.6 \mathrm{eV})$ with a variable spot size $(30-400 \mu \mathrm{m})$. Analyses of most samples are typically conducted with a $400 \mu \mathrm{m}$ X-ray spot size for maximum signal and to obtain an average surface composition over the largest possible area. The instrument has a hemispherical electron energy analyzer equipped with a 128 multi-channel detector system. The base pressure in the analysis chamber is typically $2 \times 10^{-9}$ mbar or lower. Samples were prepared for analysis by placing a drop of the liquid onto a clean glass slide. For samples that were solid at room temperature, the material was gently heated on a hot plate and a portion of the softened material was extracted and smeared onto the glass slide. Wide energy range survey spectra $(0-1350 \mathrm{eV})$ are acquired for qualitative and quantitative analysis using an analyzer pass energy of 200 $\mathrm{eV}$. For assessing the chemical bonding of identified elements, narrow energy range core level spectra were acquired with an analyzer pass energy of $50 \mathrm{eV}$. Because these samples were mounted on a glass, charging was avoided by using a charge neutralization system that uses a combination of low energy electrons and low energy argon ions for optimum charge compensation. The typical pressure in the analysis chamber with the flood gun operating was 2 x $10^{-7}$ mbar. Data were collected and processed using the Thermo Scientific Avantage XPS software package (v 4.61).

Simple atom counting was done for the 7 structures to determine a "theoretical" atomic percent (at. \%), see Table S3. Clearly, each sample should have nearly the same atomic \%, except for a loss of carbon when the dimer and trimer units are created.

Each of the samples was loaded into the XPS instrument and an initial "survey scan" was performed. Survey scans used wide energy range and checked for all elements present. In each case, $\mathrm{C}, \mathrm{O}, \mathrm{N}, \mathrm{S}$, and $\mathrm{F}$ were observed. All the samples showed some amount of Si, varying from a trace level $(\sim 0.1$ at.\%) to 4.5 at.\%. The resulting surface composition, expressed in atomic $\%$, 
is shown below in the lower part of the Table S3. Overall, the experimental data found for each sample are in the right range. Several factors could account for the small differences. First of all XPS only measures the outer 5-8 $\mathrm{nm}$ of the prepared sample, not the overall bulk composition. Second, there could be adsorbed species from the brief exposure to air as the samples were prepared for analysis $\left(\mathrm{CO}, \mathrm{CO}_{2}\right.$, hydrocarbons, water, etc.). Also, the surfaces of samples that were liquid and those that were formed by melt/solidification could have differ from the "bulk". Special attention has been paid to analysis of $\mathrm{Br}$ content. Detailed analysis showed that only two samples ( $\mathrm{n}=10$ and $\mathrm{n}=109$ ) had a small $\mathrm{Br}$ signal. In both cases, the amount of $\mathrm{Br}$ was $<0.1$ at.\% (Table S3).

Table S3: Analysis of the atomic \% of our samples: Theoretical estimates (top) and XPS experimental data (bottom)

\begin{tabular}{lrrrrrrr}
\multicolumn{2}{c}{ Atom \% } & \multicolumn{2}{c}{ Theoretical } \\
& IL-1 & \multicolumn{1}{c}{ IL-2 } & \multicolumn{1}{c}{ IL-3 } & N=10 & $\mathbf{N}=\mathbf{7 2}$ & $\mathbf{N}=\mathbf{1 0 9}$ & $\mathbf{N}=\mathbf{3 3 3}$ \\
\hline C & 44.8 & 43.9 & 44.2 & 47.3 & 45.2 & 45.1 & 44.9 \\
O & 20.7 & 21.1 & 20.9 & 19.8 & 20.6 & 20.6 & 20.7 \\
N & 6.7 & 7.0 & 7.0 & 6.4 & 6.8 & 6.7 & 6.9 \\
S & 6.7 & 7.0 & 7.0 & 7.3 & 6.9 & 6.9 & 6.9 \\
F & 20.7 & 21.1 & 20.9 & 19.2 & 20.4 & 20.5 & 20.6
\end{tabular}

Surface Compositon (at.\%) - Experimental

\begin{tabular}{|c|c|c|c|c|c|c|c|}
\hline & IL-1 & IL-2 & IL-3 & $\mathrm{N}=10$ & $\mathrm{~N}=72$ & $\mathrm{~N}=109$ & $\mathrm{~N}=333$ \\
\hline C & 45.9 & 42.9 & 49.4 & 47.4 & 43.3 & 45.0 & 48.7 \\
\hline 0 & 23.1 & 22.1 & 21.3 & 21.4 & 21.9 & 21.7 & 20.8 \\
\hline $\mathrm{N}$ & 4.7 & 5.9 & 5.0 & 5.7 & 5.8 & 5.9 & 5.6 \\
\hline S & 7.2 & 8.6 & 7.4 & 7.9 & 8.1 & 8.2 & 7.6 \\
\hline $\mathrm{F}$ & 14.7 & 20.4 & 15.1 & 17.2 & 20.8 & 18.8 & 17.2 \\
\hline Si & 4.5 & 0.1 & 1.9 & 0.4 & 0.1 & 0.5 & 0.2 \\
\hline$B r$ & 0 & 0 & 0 & $<0.1$ & 0 & $<0.1$ & 0 \\
\hline
\end{tabular}

The $\mathrm{N}$ 1s region shows two very distinct narrow peaks that are consistent with $\mathrm{N}^{+}$(cation, polymer part) and $\mathrm{N}^{-}$(TFSI part) (Figure S3). Their areas are $\sim 1: 1$ in all samples and confirm the expected structure. The results of XPS together with NMR data demonstrate that almost complete quaternization and ion exchange was achieved in all samples studied in this work. 


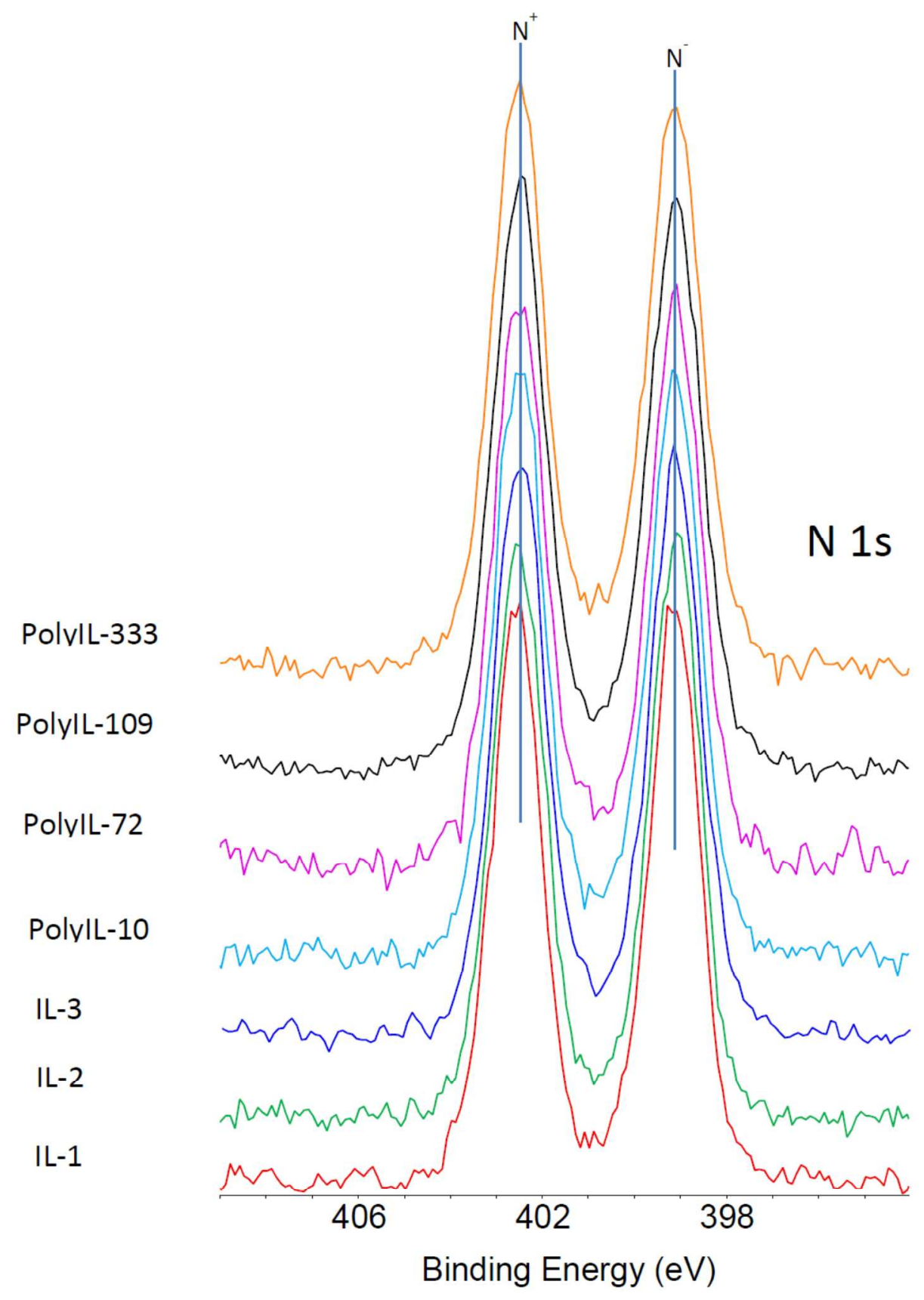

Figure S3 XPS spectra of $\mathrm{N}$ 1s region show equal area of the $\mathrm{N}^{+}$and $\mathrm{N}^{-}$peaks confirming a complete quaternization in our samples. 